\title{
Ciberespacio y mundo virtuales: el caso de Active Worlds.
}

\author{
Cyberspace and virtual worlds: \\ the case of Active Worlds.
}

Iván Infantas Barbachán**

\section{Resumen}

Las nuevas tecnologías de la información y de la comunicación, NTIC, básicamente Internet, alteran las formas de interacción social, gracias a las grandes posibilidades que actualmente ofrece el ciberespacio a las sociedades. A pesar de existir todavía una diferenciación de uso y de accesibilidad al ciberespacio, es probable que las personas, que tienen acceso a las tecnologías digitales, sientan que pueden estar 'aquí' y 'allá' de forma instantánea, lo cual causa - quizá sin percatarse, - alteraciones en las nociones tradicionales del espacio-tiempo. Una de las tantas formas de comprender el nuevo espacio emergente, es decir el ciberespacio, es mediante el estudio de los M undos Virtuales, entre los cuales se destacan los sub-mundos virtuales de Active Worlds.

Palabras clave: Active Worlds, ciberespacio; mundos virtuales; nuevas tecnologías de la información y de la comunicación. 


\section{Abstrct}

The new information and communication technologies, NTIC, basically the Internet, alter the forms of social interaction, thanks to great possibilities that cyberspace is offering to societies. In spite of differential access and cyberspace use, it is likely that individuals that have access to this digital technology, feel that they can be "here" and "there" in an instantaneous manner. The above causes, perhaps without being conscious, alterations in the traditional notions of space and time. One of the many ways to comprehend the new emerging space, that is, cyberspace, is through the study of virtual worlds, among which the sub-worlds such as Active Worlds, stand out.

Key words: Active Worlds; Cyberspace; New information and communication technologies; SW City; virtual worlds. 


\section{Introducción}

La incidencia de las Nuevas Tecnologías de la Información y la Comunicación, NTIC, en la sociedad, generan profundas alteraciones en las dinámicas sociales y territoriales; tanto así, que es posible afirmar, que nuestras nociones tradicionales de espacio y tiempo se están trastocando (Chaparro, 2004), debido a que ahora el ser humano, dado a las opciones digitales, siente que puede estar 'aquí' y 'allá' de forma casi inmediata. A pesar de haber una diferenciación en cuanto la accesibilidad a las NTIC, a la que Castells (2001) denomina como divisoria digital, y Chaparro (2007) como segregación digital, el uso de las tecnologías digitales, principalmente Internet, se viene incrementando aceleradamente. Ahora bien, es equívoco pensar en una sustitución del espacio por otro; sin embargo, sí es pertinente indicar que el espacio, que emerge de las redes de telecomunicaciones - el ciberespacio, redimensiona sustancialmente las dimensiones espacio temporales tradicionales, debido a la posibilidad de virtualizar algunas actividades sociales.

En ese sentido, el ciberespacio se presenta como un espacio experiencial dentro de un contexto social, donde el proceso de virtualización tiene un papel importante ya que abarca, desde las comunidades virtuales con intereses comunes, hasta el desdoblamiento de la personalidad en mundos digitales (Lévy, 1999), como es el caso de los submundos virtuales de Active Worlds, los cuales son entornos virtuales en tres dimensiones (3D), que poseen una geografía virtual - tanto humana como física- parecida así como diferente al mundo real, donde permiten al usuario - conocido como avatars- socializar, construir, organizar y modificar su propio territorio virtual.

Así pues, como las sociedades actualmente presencian formas poco ortodoxas de relaciones sociales y un nuevo régimen espacio temporal, producto del reciente espacio emergente denominado: ciberespacio, el presente artículo se centra en mostrar el análisis de la concepción del ciberespacio, a partir del estudio del territorio virtual y el comportamiento de los avatars de los sub-mundos de Active Worlds, específicamente el sub-mundo virtual de SW City.

Este artículo puede considerarse una síntesis del Trabajo de Grado de la Maestría en Geografía, presentado y sustentado en el Edificio del Centro de Investigación y Desarrollo en Información Geográfica, CIAF, del Instituto Geográfico Agustín Codazzi, IGAC, el 27 de noviembre de 2008. El Trabajo de Grado fue dirigido por el profesor Jeffer Chaparro Mendivelso.

\section{Metodología}

Para lograr el análisis de la concepción de ciberespacio, a partir de los submundos virtuales de Active Worlds, se realizaron principalmente trabajos de campo virtual. Sin embargo, al no existir un diseño metodológico definido para 
estudios en entornos virtuales, se emplearon técnicas de investigación utilizadas en trabajos de planificación territorial normal, las cuales, para la geografía, consisten en leer e interpretar los componentes que organizan un territorio, además de aproximarse a cómo está estructurado su funcionamiento, como una unidad orgánica.

Para realizar el trabajo de campo virtual en el territorio virtual de SW City, se aplicó la técnica de inmersión, muy usada por la antropología social y cultural, y los conceptos de la etnografía del ciberespacio. La primera consiste en establecer vínculos con otros nativos, para así observar sus actividades diarias desde dentro, y no desde fuera de su vida comunal, con el fin de comprender clara y extensivamente la vida social de la comunidad (Evans-Pritchard, 1957). La segunda, es similar a la primera, ya que la relación entre el antropólogo y el nativo sigue siendo bidireccional; la diferencia radica en que el estudio de las interrelaciones de los grupos sociales no se realiza en un espacio concreto, sino en un espacio no físico (Mayans, 2002). En todo caso, lo que se hizo fue tomar ambas perspectivas para estudiar e investigar un mundo virtual.

A través de la técnica de inmersión, el trabajo consistió en sumergirse y moverse por el entorno virtual mediante una representación digital propia, con el propósito de conocer y comprender mejor el territorio virtual de SW City. Dentro del territorio virtual de SW City, se analizó la morfología total de la ciudad virtual y el comportamiento de los avatars. Para el análisis morfológico virtual, se hizo lo siguiente: 1) en primera instancia, se describió, en forma generalizada, el entramado físico, natural y territorial de la ciudad virtual. 2) Luego, se conoció ampliamente la ocupación del suelo virtual, tanto en el aspecto macro como micro, así como también sus equipamientos estructurantes virtuales. 3) Por último, se interpretó la composición urbana del paisaje virtual de SW City; además, se espacializaron los elementos geográficos del espacio urbano del territorio virtual de SW City.

\section{L os mundos virtuales: Active Worlds (AW)}

Active Worlds es una plataforma disponible en Internet, que brinda al usuario -que dispone de ciertas tecnologíasposibilidades experienciales en entornos virtuales tridimensionales. Prácticamente, Active Worlds es un plataforma en 3D que contiene el mundo virtual de Alpha World y sus submundos virtuales (Active Worlds, 2008), el cual permite al Avatar - la proyección digital de la personainteractuar, colonizar, diseñar y construir. Por tanto, decir que sólo es un video juego o un 'chat' es equívoco, porque va más allá de eso. De hecho, es uno de los pocos sistemas multiusuarios que permite al público, además de socializar, configurar su propio entorno virtual.

Los inicios de AW se dieron en el año 1994; sin embargo, hasta el 28 de junio de 1995 se dio a conocer al público en su 
primer lanzamiento. Actualmente, su propietaria es la compañía Active Worlds Inc., que es el resultado de la unión que se produjo el 21 de enero de 1999 entre las compañías Circle of Fire Studios y Vanguard Enterprises (SW City, 2008). Hoy en día, AW cuenta con un gran número de sub-mundos virtuales diferenciales; cada uno con su propia temática y dinámica.

Ahora bien, para vivir esa experiencia virtual, es importante contar con el software, disponible en el sitio Web: www. ActiveWorlds.com, además de ciertos requisitos tecnológicos indispensables para su buen funcionamiento.
La plataforma virtual consta de tres ventanas principales: una que muestra los gráficos 3D habitadas por los avatars; otra donde se encuentra el buscador Web, y la última que sirve para comunicarse o 'chatear'. También cuenta con una barra de menús y otra de herramientas para las acciones del avatar. En todo caso, si el usuario quiere desplazarse a otro mundo virtual, sólo debe seleccionarlo con el mouse. Y si quiere ver el buscador Web, le da la opción de encendido; si desea regresar a algún territorio recorrido, hay una opción donde se muestra el historial de viajes (Börner et al., 2004) (véase la Figura 1).

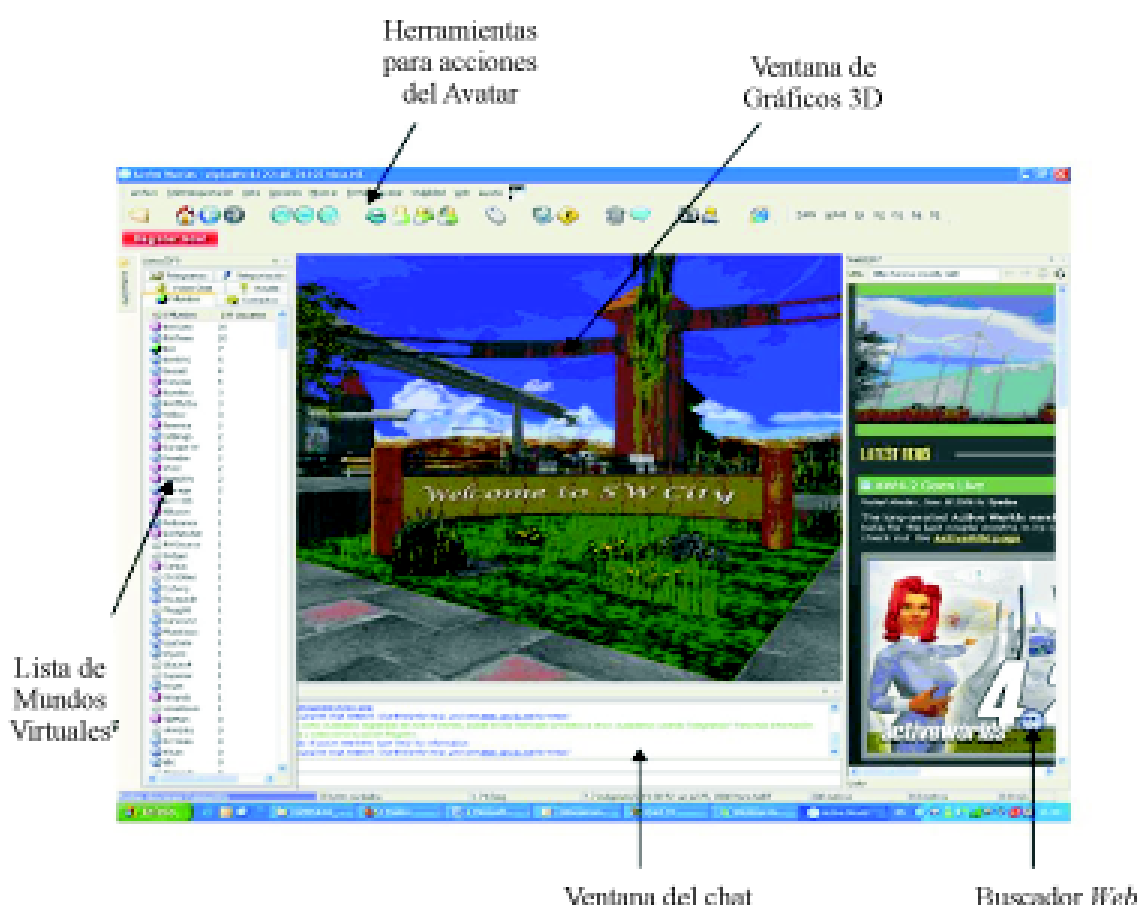

Figura 1. Plataforma virtual de Active Worlds

Fuente: imagen propia tomada de Active Worlds 


\section{Descripción del territorio virtual de SW City}

SW City es una extensa ciudad virtual que se construyó en 1999, la cual se encuentra sobre un terreno llano. Pertenece al mundo virtual de Alpha World y tiene como coordenadas de ubicación: 2217S, 3610E (véase la Figura 2). La ciudad virtual se puede presentar como una isla que se sitúa en medio de un océano virtual, llamado Irenic. Tiene aproximadamente un área de $150 \mathrm{Km}^{2}$ y está compuesta por pequeños precintos interconectados por: vías, puentes, caminos, etc.; su capital es Central City que, dicho sea de paso, es una de las más pobladas, ocupa una gran parte de la zona central de la ciudad y sus coordenadas son 2217S, 3609E. En general, no tiene una fisiografía virtual muy accidentada, aunque tiene algunas simulaciones de lomas y terrazas. Eso sí, hay una formación superficial que, comparada con el mundo real, parece una gran cordillera, la cual cubre casi todo el lado Este de la Isla y posee, aproximadamente, una altitud máxima de $254 \mathrm{~m}$.

Administrativamente, el distrito es administrado por un gobernador, que tiene a su cargo la contratación y supervisión de los gerentes del Distrito. Existen cinco distritos que conforman la ciudad; cada uno tiene un número diferenciado de precintos gestionados por gerentes.

Las actividades de los gobernantes en la Administración Municipal son varias: van desde el manejo y uso de las tierras, hasta el control de la construcción de infraestructuras. Por ejemplo, si un ciudadano quisiera adquirir parcelas y propiedades, tendría que dirigirse a la oficina de bienes y raíces, pero antes tendría que enviar un correo electrónico al funcionario, solicitando un permiso. Básicamente, se preocupan por muchas temáticas relacionadas con la creación de nuevas carreteras, la protección del medio ambiente y la construcción de obras públicas, todas ellas virtuales. También velan por la equidad entre la cantidad de tierra virtual que se da y aquella que hay disponible. Generalmente, la línea política que siguen los gobernantes es la democracia, ya que toda propuesta gubernamental se somete primero a un referéndum. 


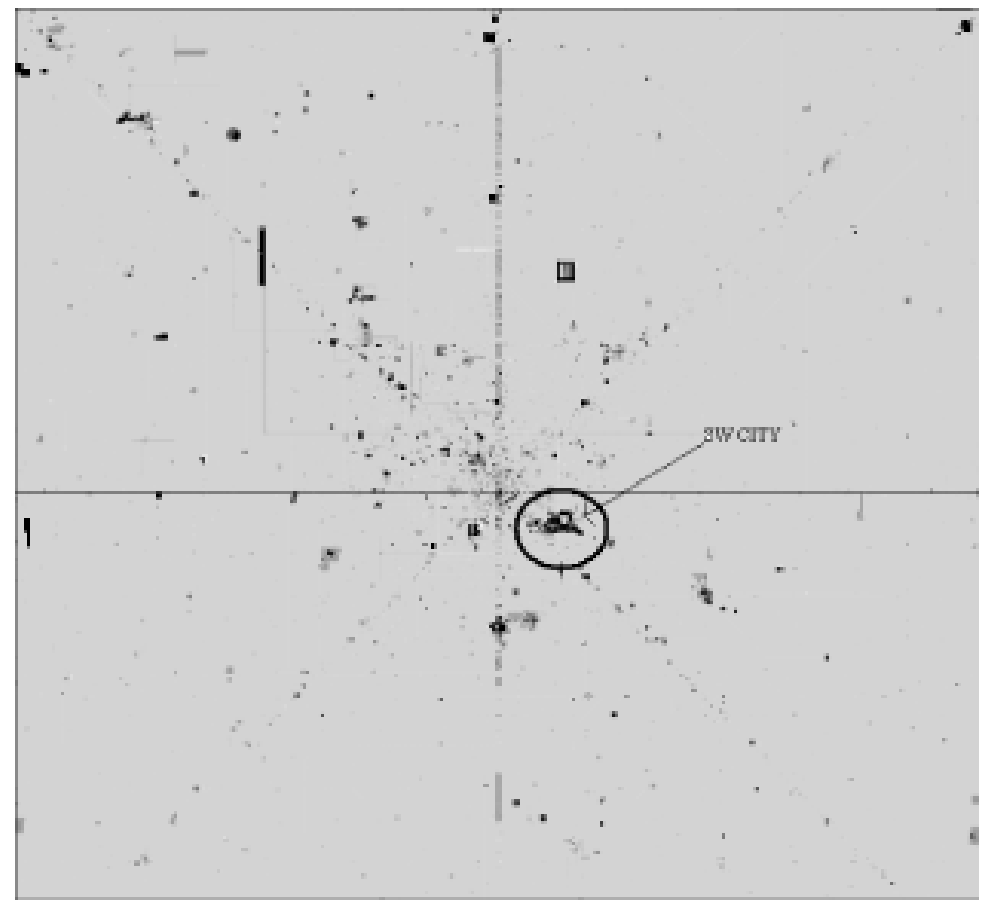

Figura 2. Ubicación de SW City en Alpha World

Fuente: modificación propia a partir de Alpha World M apper, 2008.

\subsection{M undo virtual: Alpha World}

Cómo se indicó, SW City pertenece al mundo virtual de Alpha World; pero, ¿qué es Alpha World? Alpha World, es el primer servidor de Active Worlds que cuenta con una población mayor a cualquier otro mundo virtual; además, tiene características muy semejantes al terreno físico. Esto, debido a la creación de una nueva herramienta de programación que se ejecuta en Internet, llamada VRML - Virtual Reallity $M$ arkup Language: lenguaje de marcado, de realidad virtual- (Kerckhove, 1999). $\mathrm{Su}$ superficie virtual es enorme; consta de casi $429038 \mathrm{Km}^{2}$ y es cuatro veces más grande que el estado de California. La geografía que predomina en Alpha World es simple, debido a que está cubierta, en su mayoría, por una extensa llanura (Active Worlds, 2008).

En un inicio, era un territorio completamente virgen. Ahora, este mundo es tan popular que, desde que abrió sus puertas, ha tenido más de un cuarto de millón de personas que lo han visitado (Active Worlds, 2008). Esto motivó a algunos investigadores, a espacializar y a representar en mapas, el crecimiento y desarrollo de las infraestructuras construidas por los ciudadanos, como: calles, jardines, casas, edificios, parques, etc. 
Existe un mapa elaborado a finales de febrero de 1998, que da la sensación de ser una foto tomada en la noche por el brillo que reflejan los píxeles de las áreas más urbanizadas (Dodge, 1999) (véase la Figura 3). Como se ve, gran parte del territorio de Alpha World no está del todo ocupado, ya que hay un gran porcentaje que no tiene ese brillo pixelar, el cual representa la presencia constructiva del avatar. Puede ser que la densidad haya aumentado en los últimos años; pero todavía predomina ese patrón: el de espacios vacíos. Como bien dice Dodge (1999): todavía Alpha World es muy poco poblado, en comparación con la sobrepoblación que existe en algunos países del mundo.

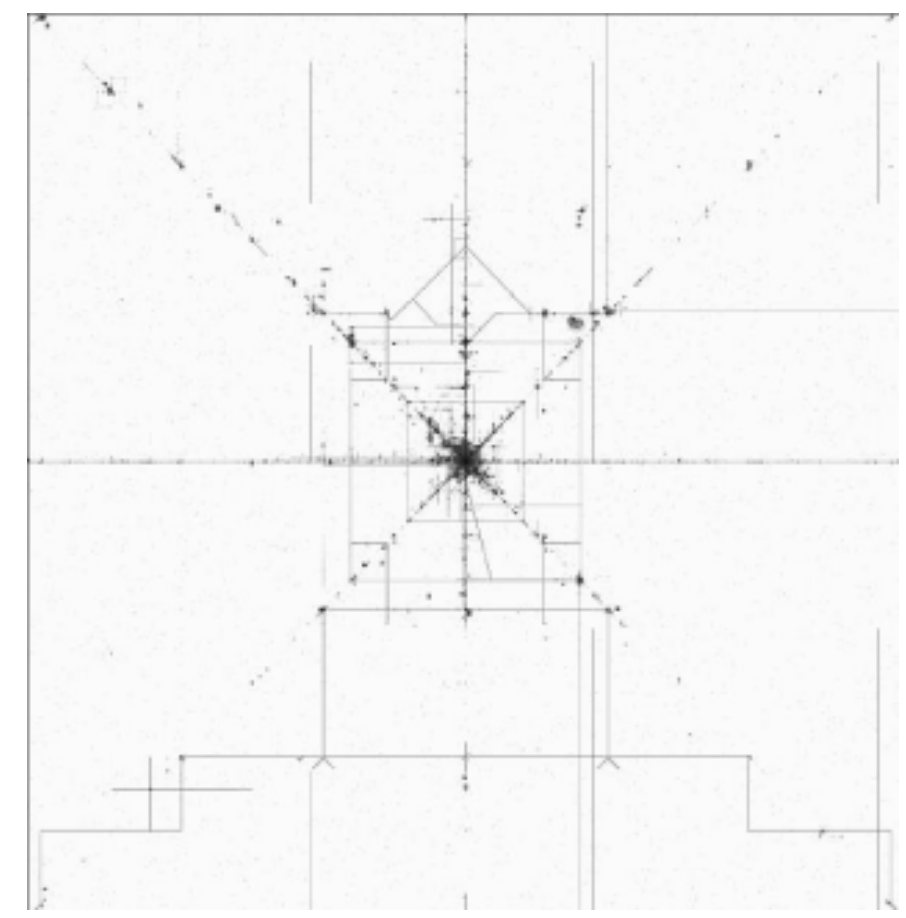

Figura 3. Densidad Poblacional de Alpha World

Fuente: Vevo, 1998, citado por: Dodge, 1999. 
Dentro de ese contexto, Greg Roelofs y Pieter Van Der Meulen crearon unas imágenes, que muestran la morfología urbana de una manera casi secuencial -diciembre de 1996, febrero de 1998 , agosto de 1999 y agosto de 200- (Active Worlds, 2008) (véase la Figura 4). El objetivo era visualizar el desarrollo de Alpha World, expresado en sus infraestructuras. En efecto, el crecimiento se aprecia, tanto así que se observa claramente cómo la concentración céntrica se expande y diluye poco a poco el dibujo de la estrella. Roland Villet - uno de los programadores de Alpha World- dice que la gran impresión que le dejaron esos mapas, fue el gran parecido que tienen con los mapas reales, en el sentido que se percibe una complejidad $\mathrm{y}$ un desorden en el crecimiento (Dodge, 1999).

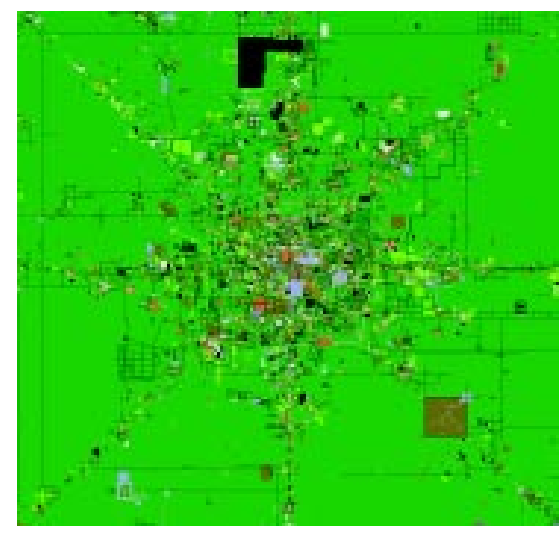

Diciembre de 1996

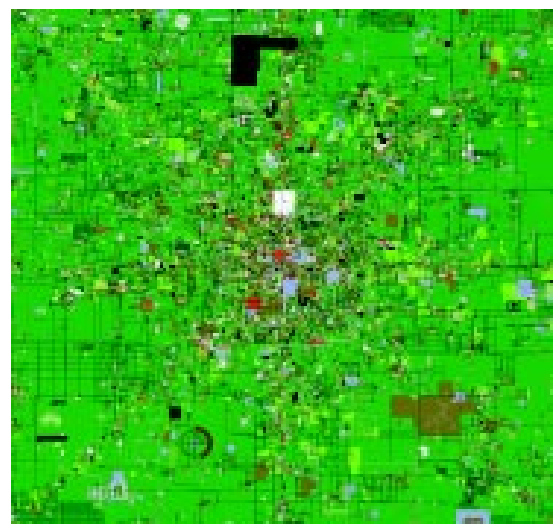

Agosto de 1999

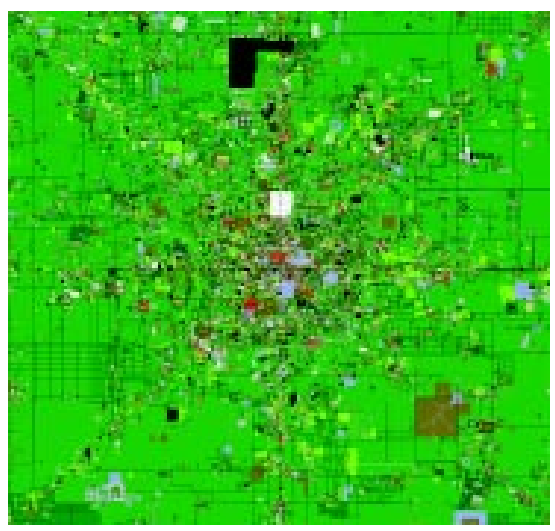

Febrero de 1998

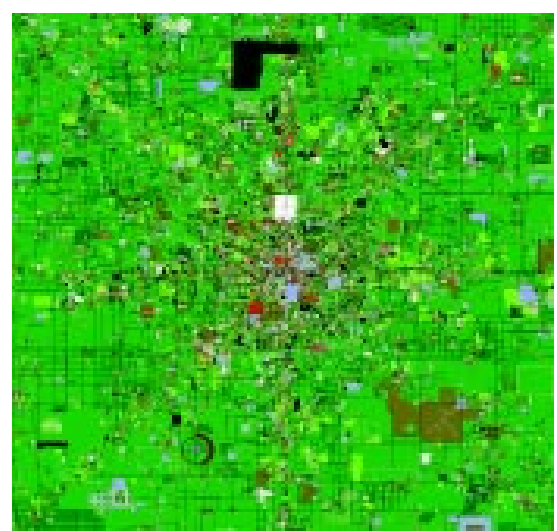

Agosto de 2001

Figura 4. Configuración territorial del mundo virtual de Alpha World Fuente: Active Worlds, 2008. 
Active Worlds está basado en un sistema de coordenadas cartesianas. Todos sus mundos virtuales, incluido Alpha World, tienen como punto de origen: $(0,0)$; el cual es conocido como Ground Zero (GZ) y, básicamente, es la puerta de entrada por defecto. El GZ sirve como referencia de ubicación al usuario; por ejemplo, si un ciudadano introduce una dirección, lo haría de la siguiente manera: $(67 \mathrm{~N}, 42 \mathrm{~W})$; esto significaría que se encuentra a 670 metros al Norte y a 420 metros al Oeste del GZ. Lo más curioso de esto es que ya los ciudadanos no dan las direcciones de sus viviendas, como suelen hacerlo en el mundo real, con el número y el nombre de la calle, sino que lo dan en coordenadas (Dodge, 1999).

Ahora bien, los caminos son intermitentes y discontinuos, no hay una estructura integradora que articule los sub-mundos virtuales que conforman el mundo virtual de Alpha World; es decir, no hay una estructura que relacione la estructura global con las locales, situación que difiere de SW City que sí tiene un equipamiento estructurante, que intercomunica y conecta los distritos y precintos que pertenecen al territorio virtual; esto, gracias a la presencia de carreteras y medios de transporte que colaboran con esa conectividad espacial, aunque todavía se aprecian espacios virtuales vacíos.

¿Es importante la conectividad espacial para la existencia de los avatars? Se cree que no. El avatar puede desenvolverse tranquilamente sin depender de dicha integralidad; ni siquiera el desarrollo de los sub-mundos virtuales se somete a la articulación de los territorios virtuales. Este es un fenómeno socio-espacial distinto, si se compara con nuestro planeta, donde el proceso de globalización y los lazos económicos, sociales y políticos, se acrecientan cada vez más.

\section{Resultados}

\subsection{A vatars: una experiencia virtual en el Sub-M undo de SW city}

Para mejor entendimiento, es importante definir el término avatar. Según la Real Academia Española, el término avatar se define de la siguiente manera: "(Del fr. avatar, y este del sánscr. avatâra, descenso o encarnación de un dios). $m$. Fase, cambio, vicisitud. U. m. en pl. || 2. En la religión hindú, encarnación terrestre de alguna deidad, en especial Visnú. || 3. Reencarnación, transformación" (RAE, 2008). Hay otras definiciones, las cuales afirman que es: "una máscara digital que uno se pone para identificarse y adoptar una actitud, en pantalla completa, en los nuevos entornos 3-D creados en línea" (Kerckhove, 1999).

El término avatar proviene de la mitología hindú; así era la manera como se llamaba al Dios que se daba vida a sí mismo en la tierra (Spring, 2000). Las representaciones que el avatar puede adquirir son variadas: aves, reptiles, insectos, pescados, pingüinos, lobos, hadas, frutas, objetos, dinosaurios, 
humanos, alienígenas, árboles, autos, cosas, monstruos, etc.; su apariencia depende mucho de la escogencia de la persona (véase la Figura 5).

En AW existe una lista de Avatars, disponible para los registrados; para los usuarios que no son ciudadanos, sólo hay un avatar con vestimenta de turista, como en el presente caso, tal como puede verse en la Figura 6, ya que para ser residente hay que pagar mensualmente una suscripción. Hay que indicar, así mismo, que hay avatars personalizados que, normalmente, son copias del mismo usuario.

Durante la exploración en SW City, se observó que el avatar puede desplazarse caminando o volando; además, puede charlar y/o comunicarse con otros individuos, gracias a una ventana estilo 'chat', donde se introduce el texto deseado.
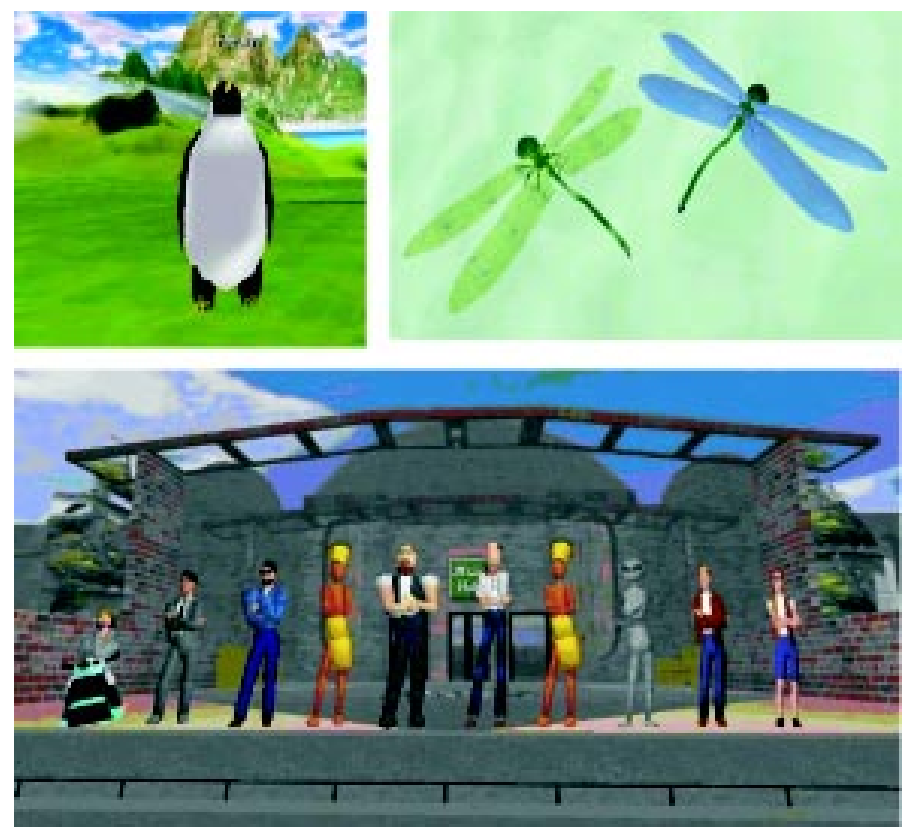

Figura 5. Algunas representaciones digitales de los usuarios -AvatarsFuente: SW City, 2008. 


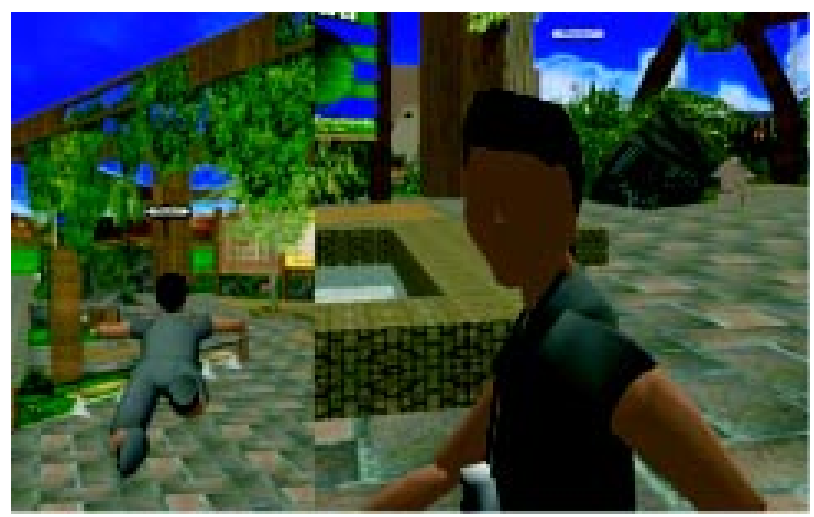

Figura 6. Representación digital propia con vestimenta de turista Fuente: imagen propia del territorio virtual de SW City, 2008.

$\mathrm{Al}$ entrar a un mundo virtual, en este caso al territorio virtual de SW City, se aterriza en un lugar que, básicamente, es el centro de la ciudad. En SW City no se encontró una gran cantidad de avatars y, aunque se quiso entablar conversaciones con algunos individuos, el idioma lo dificultó ya que, la mayoría, por no decir todos, hablan inglés, y no necesariamente que se pueda entender con facilidad, debido a la gran cantidad de abreviaturas y jergas que utilizan para comunicarse. A pesar de que se puedan encontrar sub-mundos virtuales en español, los entornos virtuales que más predominan son de habla Inglesa.
Existen cabinas que, de manera inmediata, teletransportan a los avatars a tierras virtuales lejanas; proceso que también pueden hacerse mediante la colocación de coordenadas (véase la Figura 7). Además del vuelo, estas formas de viaje son útiles para explorar el territorio virtual de SW City. En todo caso, cuando se habla de las principales formas de desplazamiento en SW City, y en general en todos los sub-mundos virtuales de Active Worlds, se puede mencionar las siguientes: caminando que es la más tradicional-, volando y teletransportándose. 


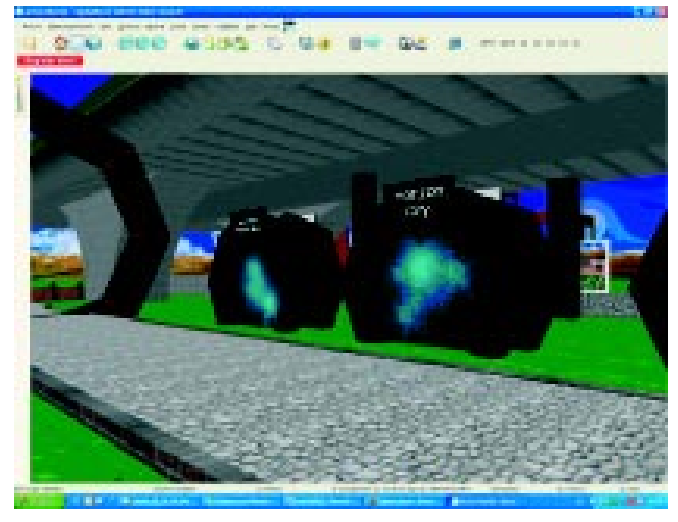

Figura 7. Puertas de teletransportación de SW City

Fuente: imagen propia del territorio virtual de SW City, 2008.

En SW City, así como en otros territorios virtuales, los usuarios tienen la posibilidad de conocer gente y de construir casas, edificios, etc. en 3D, en el ciberespacio. Ahora bien, sólo aquellos ciudadanos que cumplan ciertas especificaciones de AW, tienen la oportunidad de apropiarse de terrenos virtuales disponibles para crear su propio entorno.

El ser ciudadano certificado da la oportunidad de poder construir estructuras tridimensionales y de reclamar tantas parcelas virtuales como se desee; situación reflejada con la localización de objetos. Cuando el usuario haya colocado el objeto sobre su territorio virtual, es difícil que sea desalojado o movido por otra persona. Además, al ser dueño de la propiedad, el residente puede distribuirlo, duplicarlo y hasta ponerlo a la venta o alquilarlo (Grané et al., 2007).

\subsection{Composición urbana del paisaje virtual de SW city}

En el entorno virtual de SW City, existen varias formas de relieve virtual. Se ven nevados, dunas, playas, bosques y terrazas, entre otros (véase la Figura 8). Pero, sin duda, la que mayor presencia tiene son las llanuras. Cada una de ellas guarda ciertas características físicas y bioclimáticas muy particulares que, de alguna forma, profundiza la sensación de inmersión del usuario. Ahora bien, si se habla de su paisaje virtual, se puede decir que SW City es como una colcha de retazos que combina una serie de elementos que organiza el territorio. En él, se aprecian espacios que tienen que ver con zonas de tipo industrial, militar, turística, deportiva, comercial, recreacional, sociocultural, administrativa y habitacional (véase la Figura 9). 


\section{color}

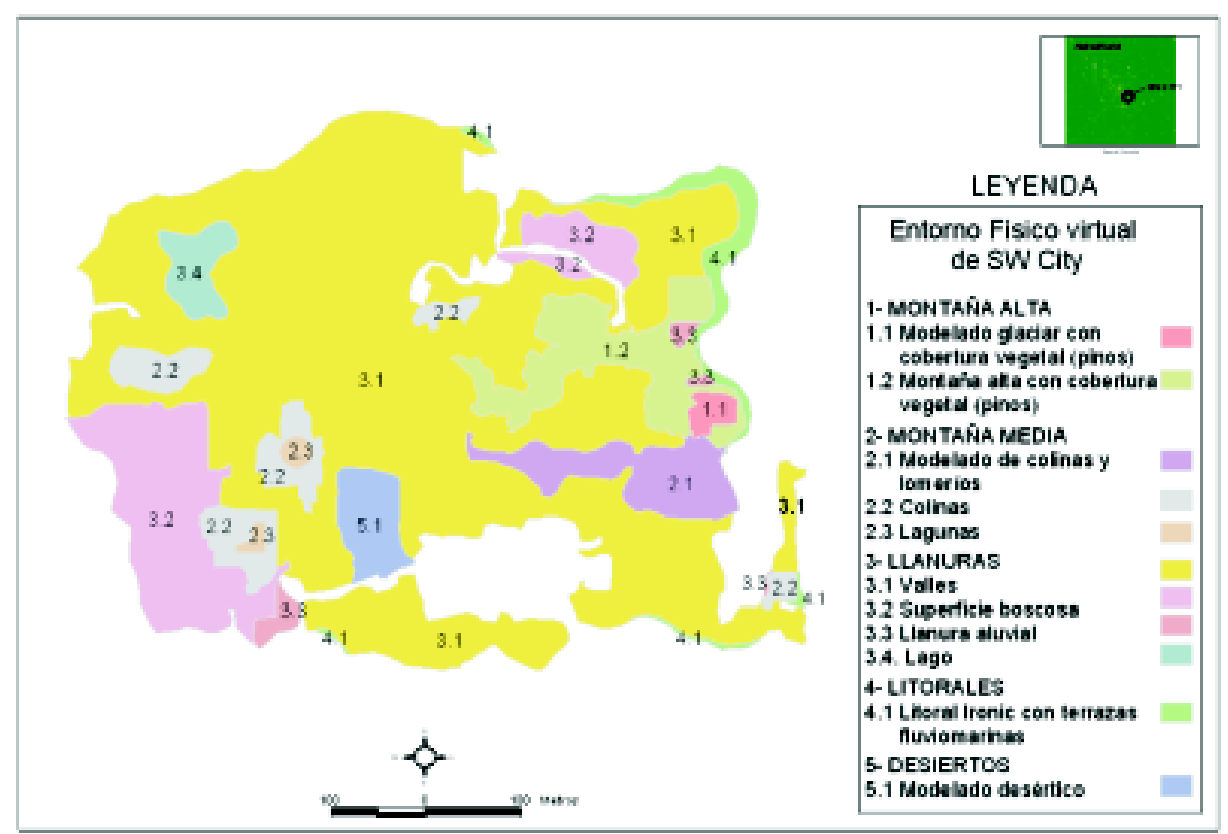

Figura 8. Entorno físico virtual de SW City

Fuente: modificación propia a partir de Alpha World M apper, 2008.

Sin embargo, no se aprecian entornos socio-espaciales virtuales rurales, que tengan como principal actividad la agricultura y la ganadería. A pesar que en SW City hay grandes extensiones de terreno virtual disponible, no se encuentran formas de cultivo, ni ningún tipo de plantación virtual. Tampoco se vislumbran centros ganaderos, ni áreas virtuales para el pastoreo.

En SW City no hay una diferenciación entre lo urbano y lo rural, porque, prácticamente, lo segundo no se presenta en la configuración territorial de la ciudad virtual. Es probable que los usuarios, que hacen su arribo a los mundos virtuales, sean citadinos y, por ser habitantes y/o residentes de las zonas urbanas, desconocen otro tipo de modelos, o no están familiarizados con otras costumbres y comportamientos no urbanos. O incluso, tal vez no les interesan las actividades agropecuarias $\mathrm{y}$, por tanto, no las representan en el mundo virtual. 


\section{color}

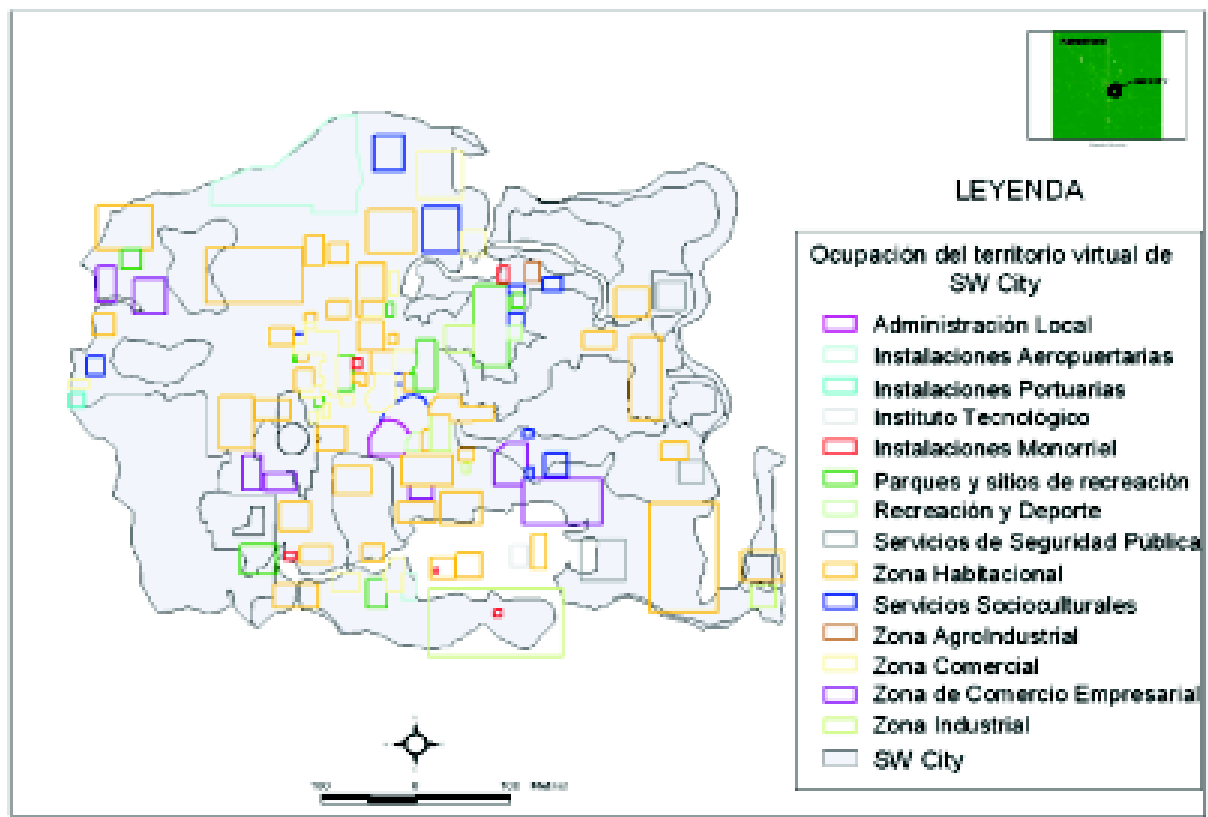

Figura 9. Ocupación del territorio virtual SW City

Fuente: modificación propia a partir de Alpha World M apper, 2008.

Poblacionalmente hablando, la ciudad virtual tiene una densidad muy desigual. Como no hay censos, por ende ningún dato estadístico, la densidad poblacional se dedujo a partir de la densidad de construcción (a mayor densidad de construcción en un espacio de uso del suelo "vivienda", corresponde mayor cantidad de población). Entonces, se observa, que el centro de la ciudad presenta una mayor concentración de infraestructuras mayor al resto, como puede verse la Figura 10. 


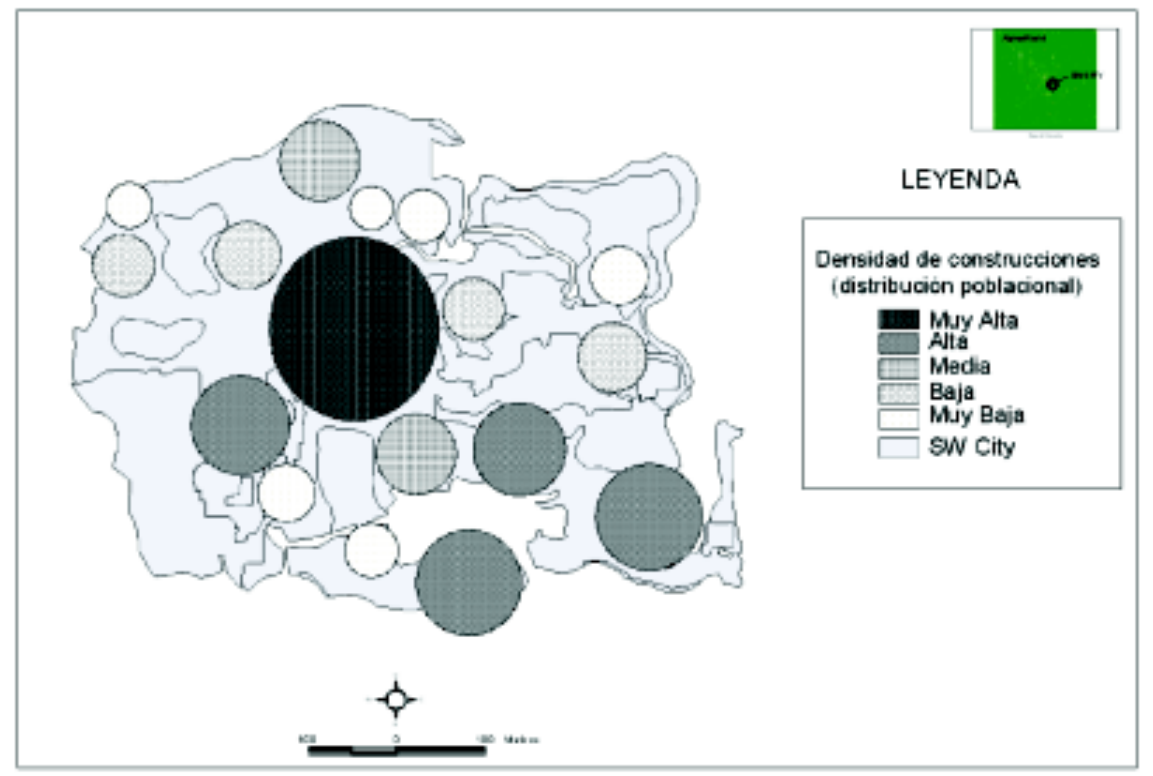

Figura 10. Ocupación del territorio virtual SW City

Fuente: modificación propia a partir de Alpha World M apper, 2008.

Quizá esa mayor densidad en el centro se deba a que, en un inicio, cuando SW City era un espacio virtual virgen, los avatars empezaban a ocupar esa zona, porque básicamente era y es la puerta de entrada por defecto. Es decir, cuando el usuario inicialmente hace su ingreso a SW City, aterriza de forma instantánea en el centro de la ciudad; esto posiblemente llevó a que los ciudadanos empezaran allí a construir parques, viviendas, casas y edificios, lo cual generó una alta concentración de infraestructuras, que en el resto de la ciudad virtual no se ve.

Es más, siguiendo casi los mismos patrones urbanos, la mayoría de edificios que dominan la zona concéntrica de la ciudad virtual pertenecen a entidades administrativas locales y privadas. En ese sentido, lo que propicia estas situaciones es una distribución espacial muy heterogénea de SW City.

Ahora bien, las posibilidades que existe de realizar diferentes formas de construcción son muy altas. Se observan arquitecturas muy sofisticadas, simples, rústicas y, en algunos casos, muy poco ortodoxas, ya que, así como se pueden distinguir viviendas y urbanizaciones clásicas, también se vislumbran edificaciones modernas, complejas y, algunas, bastante lujosas; además de otras muy creativas. $Y$ es que en el ciberespacio se puede o no seguir la misma lógica constructiva de las ciudades físicas. Aunque, en general, se puede señalar que existe un fuerte traslado de los patrones estructurales del mundo real. 
Es importante resaltar que las formas espaciales producto de la pobreza, la marginación, la discriminación y la segregación, que se dan en el mundo real, no se aprecian en el mundo virtual. Es posible que los usuarios, al estar dentro de los mundos virtuales, sólo deseen alejarse de las problemáticas reales. Es por ello que no se distingue una marcada diferenciación socioeconómica entre los avatars.

Sin embargo, sí hay replicas y patrones reales que se encuentran en la ciudad virtual, como el estructuramiento interno que posee (véase la Figura 11). Se observan diferentes zonas para cada tipo de actividad. Por ejemplo, el centro de la ciudad es una zona exclusivamente urbana, donde resaltan las infraestructuras relacionadas con la vivienda, el comercio, la administración local y los negocios. En la periferia de la ciudad, en cambio, se asientan zonas vinculadas a las instalaciones aeroportuarias, portuarias y a la industria. La distribución y la forma de organización es tal, que no se entrecruzan las zonas más predominantes en cuanto el uso. En ese sentido, cada actividad tiene su propio espacio virtual, lo cual denota una interesante diferenciación funcional del territorio virtual.

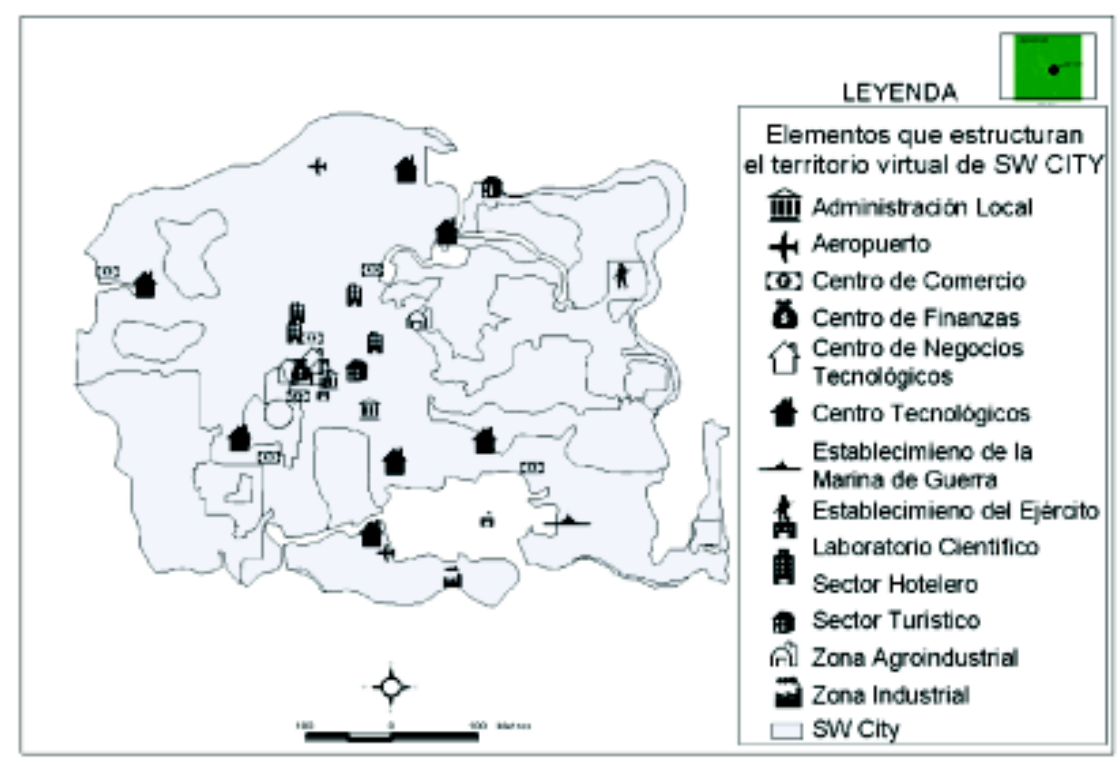

Figura 11. Elementos estructurantes del territorio virtual SW City Fuente: modificación propia a partir de Alpha World Mapper, 2008. 
Ahora bien, el que haya varios sectores ocupados, unos más que otros, no significa que por las principales calles o avenidas se vea una circulación constante de avatars con quienes se pueda charlar o entablar una conversación. O que se perciba avatars trabajando en alguna industria, administración local, hotel, banco o compañía. En la lógica de SW City no se observa una dinámica de trabajo como la del mundo real. No es común ver, en el día a día, un avatar realizando labores pesadas, ni atendiendo a otro avatar en una ventanilla de un banco, ni verlo sentado en una oficina discutiendo problemas administrativos, políticos, ni económicos con otro avatar.

Lo que sí se encuentra, son personajes que cumplen el papel de 'maniquí', con la salvedad de que ellos tienen un diálogo definido con preguntas y respuestas programadas por los usuarios, posibilitadas por unas aplicaciones antes comentadas-denominadas Bots. Hay varios Bots que se encuentran en SW City.

Normalmente, cuando se entra a una tienda, a un restaurante, a un bar o a los centros administrativos, los Bots prestan servicios informativos, pero no son muy dinámicos. Los usuarios crean estos personajes con el fin de definir una atmósfera de animación y, de alguna manera, para que se sienta que el avatar que está explorando la ciudad virtual no está sólo. Que no haya un buen número de peatones desplazándose o laborando, no implica dejar de asumir que SW City es considerada como una de las principales ciudades virtuales de Alpha World.

Finalmente, se puede decir, a partir de los patrones reales encontrados en el mundo virtual, que el estar en el ciberespacio no significa estar desconectado de la realidad, porque no se puede olvidar que quien está detrás de ese personaje virtual es un ser humano, el cual, así como puede plasmar sus imaginarios en entornos virtuales también refleja, como ser social que es, sus deseos, percepciones y vivencias construidas en el mundo real.

Los usuarios no se liberan totalmente de la realidad; aunque, como se mencionó, sí lo hacen de muchas de las problemáticas que se frecuentan en la mayoría de las ciudades reales. Por ejemplo, en SW City no se ve a indigentes dormir en el parque, ni niños, ni adultos pedir limosna. No se ven avatars comiendo sobras de alimentos tiradas en la calle. No se perciben problemas de basura, ni contaminación industrial y vehicular. No existe el caos vehicular, ni las dificultades de ir de un lugar a otro con la premura del tiempo. Prácticamente SW City, al no tener ese tipo de conflictos, de alguna manera se percibe como una ciudad utópica, de ensueño y eso hace también -así no se crea-, muy complejo y muy complicado de abstraerlo y comprenderlo, porque es un híbrido, donde se conjuga lo real con lo irreal; sólo ello ya dificulta su entendimiento. 


\section{Conclusiones}

Los mundos virtuales son como una realidad paralela, diferente $\mathrm{y}$, a la vez, parecida a la nuestra, porque existe un traslado de ciertos patrones territoriales que contribuyen con el crecimiento y la configuración del territorio virtual, en donde es probable que ese traslado se deba a que el usuario no conoce -o imagina - otro estilo de vida, ni otros modelos de ciudad.

Ahora bien, abstraer un territorio virtual, en este caso SW City, no es un trabajo fácil, sobre todo cuando se busca comprender la dinámica socio-espacial, porque, primero, buena parte del territorio virtual se encuentra deshabitado - sólo hay un número pequeño de avatars que no cubren la totalidad de la ciudad virtual y unos Bots programados por los usuarios-; pareciera más bien un 'pueblo fantasma' Segundo, porque a los poquísimos ciudadanos y turistas presentes, no fue nada sencillo hacerles un seguimiento, puesto que, normalmente, el avatar no se encuentra todo el día en la ciudad virtual; eso, sumado al poco manejo del idioma específico o argot, que lo hizo más complejo aún.

En ese contexto, se puede plantear que los mundos virtuales son un territorio incógnito donde, de antemano, no se puede prever lo que sucederá, más aún cuando es la primera vez que se hace un estudio del territorio virtual de SW City; es como si fuera una selva virgen, donde se hace lo posible por interpretar, para alcanzar los objetivos, pero que se truncan por lo impredecible del lugar. En ese sentido - guardando las distancias-, así como el espacio geográfico es complejo de abstraer, los entornos virtuales también lo son, porque representan otra realidad, otro modo de comportamiento muy poco ortodoxo.

A pesar de las complejidades, y las semejanzas y diferencias con el mundo real, los mundos virtuales son un producto de la sociedad, que si bien se encuentran en un espacio etéreo, son territorios virtuales concretos, susceptibles por ser explorados y descritos. Prácticamente, se presentan como 'otro espacio geográfico', como otra realidad paralela que cuenta con comportamientos socio-espaciales muy interesantes de ser abordados.

A partir de la investigación del territorio virtual de SW City, se llegó a la conclusión que el ciberespacio es un espacio no tangible, que se encuentra en el límite de lo físico y lo virtual, lo cual hace posible que se pueda combinar lo irreal y lo real. El ciberespacio permite conjugar imaginación, creatividad y realidad. Quizá las personas que se adentran al ciberespacio sólo desean como se percibió en el territorio virtual- desconectarse o alejarse del caos y de las problemáticas de nuestro mundo. En el ciberespacio se puede ser quien se desee ser. La identidad dentro de un entorno virtual se puede trastocar; en la mayoría de los casos, nadie conoce 
al otro, como es el caso de los avatars que, usualmente, tienen un sobrenombre, el cual sirve como una máscara que facilita al usuario alterar su 'yo', si así lo quiere.

En general, el ciberespacio es un entorno digital relacional, resultado de la conjugación hombre-máquina, el cual se puede asumir como un espacio híbrido, donde tanto la sociedad como las tecnologías juegan un papel importante. Ahora, si bien la tecnología es clave en cuanto el funcionamiento, desde el punto de vista personal, el ciberespacio no sería ciberespacio sin la presencia del hombre, porque es a través de sus acciones, sentimientos y pensamientos, que se le da sentido y vida. El ciberespacio no es, entonces, asunto únicamente de computadores e Internet.

Desde nuestra perspectiva, el ciberespacio se ha convertido en un nuevo entorno de interacción, donde el hombre no sólo puede intercambiar opiniones, difundir información, comercializar y navegar por sitios Web, sino que, incluso, puede crear mundos, territorios y personajes que, aunque no sean tangibles, resultan interesantes porque reflejan nuestras percepciones y nuestras fantasías. Por eso nos atrevemos a decir que, actualmente, se está desarrollando, y se seguirá haciendo, un espacio paralelo, un nuevo espacio virtual que, así como brinda muchas posibilidades, es muy complejo de comprender. Si hasta ahora dentro de la geografía existen diversos intentos por refinar sus teorías con respecto al espacio geográfico, qué se puede esperar de un tipo de espacio híbrido, que recién emerge y que combina lo real con lo irreal, lo utópico, los sueños y los imaginarios. 


\section{Literatura citada}

ActiveWorlds. 2008. Newburyport (Massachussets, U. S. A.): ActiveWorlds Inc. [En línea]. $<$ http://www.activeworlds.com/ > . Se tuvo acceso en septiembre de 2008.

Börner, K.; Hazlewood, W. y Sy-Miaw, L. 2004. Visualizing the Spatial and Temporal Distribution of User Interaction Data Collected in Three-Dimensional Virtual Worlds. [En línea]. USA: Indiana University. <http://vw.indiana.edu/cive02/ 004 borner_VisSpatialTemporal.pdf $>$. Se tuvo acceso en julio de 2008.

Castells, M. 2001. La galaxia Internet. Barcelona: Plaza \& Janés.

Chaparro, J. 2004. Sociedad y posibilidades tecnológicas emergentes. Bogotá, D.C.: Universidad Nacional de Colombia, Departamento de Geografía, Cuadernos de G eografía (13): 59-79.

Chaparro, J. 2007. La segregación digital en contexto. Revista electrónica de recursos en Internet sobre Geografía y Ciencias Sociales. Ar@cne. [En línea]. Barcelona: Universidad de Barcelona, (95). http://www.ub.es/geocrit/ aracne-095.htm. Se tuvo acceso en agosto de 2008.

Dodge, M.1999. Explorations in Alpha World: The Geography of 3-D Virtual Worlds on the Internet. [En línea]. London: University College London. Centre for Advanced Spatial Analysis, (99). < http://www.casa.ucl.ac.uk/martin/ibg99.pdf > .

Evans-Pritchard, E. 1957. Antropología Social. Buenos Aires: Nueva Visión.

Grané, M. ; Frigola, J. y Muras, M. 2007. Second Life: Avatares para aprender. [En línea]. Barcelona: Universidad de Barcelona. < http://www. utn.edu.ar/aprobedutec07/docs/ 62.pdf $>$. Se tuvo acceso el 20 de julio de 2008.

Kerckhove, D. 1999. Inteligencias en Conexión. Hacia una sociedad de la Web. Barcelona: Gedisa.

Lévy, P. 1999. ¿Qué es lo virtual? Barcelona: Paidós.

Mayans, J. 2002. Nuevas Tecnologías, Viejas Etnografías. Objeto y método de la antropología del ciberespacio. Revista Textos de la Cibersociedad. [En línea]. Observatorio para la Cibersociedad, 23. < http://www.cibersociedad. net/ archivo/articulo.php?art=23>. Se tuvo acceso el 3 de septiembre de 2008. 
Real Academia Española. Diccionario de la Lengua Española. XXII Ed. Tomo 1. Madrid: Espasa Calpe S.A., 2001.

Spring, T. 2000. Review: ActiveWorlds offers 3-D Interaction. CNN technology computing . < http://archives.cnn.com/2000/TECH/computing/09/12/3d.homesteads.idg/ > . Se tuvo acceso el 20 de julio de 2008 .

SWCity. 2008. SwCity Online :Sintax. [En línea]. < http://www.swcity.net/ > . Se tuvo acceso el 15 de marzo de 2008. 